\title{
INDUCCIÓN IN VITRO DE LA REACCIÓN ACROSÓMICA CON HEPARINA EN SEMEN CONGELADO DE TOROS BRAHMAN Y GYR
}

\section{IN VITRO INDUCTION WITH HEPARIN OF ACROSOME REACTION IN FROZEN BRAHMAN AND GYR BULLS SEMEN}

\author{
José Montes ${ }^{1}$, María Torres ${ }^{2}$, Clara Rugeles ${ }^{3}$, Roberto Almanza ${ }^{4}$, José Guimarães ${ }^{5}$
}

${ }^{1}$ MVZ, estudiante de maestría, Universidad Federal de Viçosa, Brasil, zecave@live.com; ${ }^{2}$ MVZ, estudiante de maestría, Universidad de Córdoba, Colombia, torresmariaelena2@hotmail.com; ${ }^{3}$ MVZ, M.Sc. Profesora Universidad de Córdoba, Facultad de Medicina Veterinaria y Zootecnia, Colombia, crugelespint@yahoo.es; ${ }^{4}$ MVZ, profesional particular, Colombia; ${ }^{5}$ MV, Ph.D. Profesor Universidad Federal de Vicosa, Brasil, jdguima@ufv.br

Rev. U.D.C.A Act. \& Div. Cient. 15(2): 431 - 436, 2012

\section{RESUMEN}

El objetivo de este trabajo fue evaluar la respuesta del semen congelado-descongelado de toros Brahman y Gyr a la inducción de la reacción acrosómica in vitro con heparina y su uso como prueba complementaria, para predecir la fertilidad del semen congelado-descongelado. Fueron usados siete toros cebuínos (4 Brahman y 3 Gyr), sexualmente maduros. En la evaluación seminal posdescongelación, se evaluó la Motilidad Progresiva Individual Rápida (MPIR), Anormalidades Primarias (AP), Anormalidades Secundarias (AS), Anormalidades Totales (AT) y porcentaje de inducción de la Reacción Acrosómica (RA). La RA fue determinada sobre extendidos con eosina-nigrosina, mediante microscopía óptica a 100X. No hubo diferencia entre las dosis seminales de ambas razas para la MPIR, morfología espermática y RA ( $p>0.05$ ), con excepción de las AP, donde la raza Brahman evidenció menor porcentaje de defectos que la raza Gyr ( $p<$ 0.05). El valor obtenido para las Tasas de No Retorno (TNR) fue de 79,4 1,4\%; sin embargo, no se observó asociación entre la evaluación de calidad seminal (MPIR, AP, AS, y RA) con las TNR de los toros $(p<0.05)$. Los resultados de este estudio indican que la técnica de inducción de RA puede ser empleada como una prueba complementaria en la evaluación de rutina del semen congelado bovino; no obstante, de forma aislada no es eficaz en predecir el potencial fecundante del semen criopreservado.

Palabras clave: Acrosoma, inductor, capacitación, fertilidad.

\section{SUMMARY}

The aim of this study was to value the in vitro induction by heparin of acrosome reaction in frozen semen from
Brahman and Gyr bulls, as a method to estimate frozen semen fertility. Seven sexually mature Zebu bulls (4 Brahman and 3 Gyr bulls) were used in this study. In a post-thawing seminal assessment the progressive rapid individual motility (MPIR), primary abnormalities (AS), secondary abnormalities (AP), total abnormalities (AT) and percentage of acrosome reaction induction (RA) were studied. Acrosome reactions were determined on a glass microscopic slide whith eosinenigrosine stain and examining under 100X magnification, using optical microscopy. There was no difference between the seminal doses for both races in the MOTP, spermatic morphology and RA $(p<0.05)$, however AP made an exception, indicating that the Brahman type presented a lower percentage of defects than the Gyr type ( $p>0.05)$. No correlation was observed between semen quality assessment (MPIR, AS, AP, AT and RA) and the non-return rate of the bulls $(p<0.05)$. The results indicate that $R A$ technique can be used as complementary test in the seminal routine assessment of frozen bovine semen; however, alone it is no effective in predicting the fertilizing potential of cryopreserved semen.

Key words: Acrosome, inductor, capacitation, fertility.

\section{INTRODUCCIÓN}

El análisis de calidad seminal representa una valiosa herramienta para evaluar el potencial fecundante de los machos de diferentes especies y complementa la valoración física del animal (Bissonnette et al. 2009). En programas de inseminación artificial, como en los programas de selección y de mejoramiento genético de los reproductores, la identificación de los toros fértiles es fundamental y, por ello, se aplican distintos métodos, que sugieren correlación positiva con el criterio de fertilidad del semen (Ax \& Lenz, 1986; 
Bissonnette et al. 2009). Tradicionalmente, la movilidad, la concentración y la morfología espermática han sido los parámetros más usados dentro de la evaluación de la calidad seminal (Sutkeviciene et al. 2009); sin embargo, el valor predictivo de la fertilidad es limitado, debido a la complejidad del espermatozoide y del proceso de fecundación (Costa et al. 2010).

La constante búsqueda de una prueba altamente correlacionada con la fertilidad de los reproductores, ha permitido tener a disposición distintas alternativas para medir la calidad seminal, a pesar que no exista una única técnica capaz de predecir, con gran precisión, la fertilidad de un toro (Rodríguez-Martínez et al. 1997). Las técnicas de laboratorio, para confirmar la calidad espermática, incluyen parámetros relacionados con la integridad de estructuras, como el acrosoma, la membrana celular, la mitocondria, el DNA genómico y parámetros dinámicos, como la movilidad hiperactivada, así como la interacción entre el espermatozoide y el ovocito (Bissonnette et al. 2009). La variabilidad en los resultados puede estar relacionada con los diversos factores que intervienen en el proceso de fecundación (Hammerstedt et al. 1990), como es el caso de la capacitación espermática y la reacción acrosómica (RA). Durante la capacitación espermática, los espermatozoides son activados por moléculas del aparato reproductivo de la hembra, adquiriendo un batido del flagelo más vigoroso y una mayor amplitud de desplazamiento lateral de la cabeza (Yanagimachi, 1994; Olivera et al. 2006). Después del proceso de capacitación, el espermatozoide llega a la zona pelúcida y experimenta la RA, caracterizada por la exocitosis de las enzimas hidrolíticas contenidas en el acrosoma, que facilitan la penetración del ovócito por el espermatozoide (Parrish et al. 1988; Yanagimachi, 1994).

Estos eventos son desencadenados in vitro, mediante la adición de agentes capacitantes, como la heparina (Yang et al. 1993), que producen efectos químicos, desestabilizando y potencializando el espermatozoide, la fusión de las membrana y la RA (Miller et al. 1990; Risopatrón et al. 2005). La heparina es un glicosaminoglucano (GAGs) presente en el fluido oviductal, que estimula la capacitación espermática y, a medida que se acerca la ovulación, su concentración aumenta. Además de inducir la capacitación de los espermatozoides, la heparina mejora las tasas de fecundación in vitro en bovinos (Gliedt et al. 1996; Méndez et al. 2003). Según Ax \& Lenz (1986), los espermatozoides de toros con alto índice de fertilidad tienen más RA en respuesta a sustancias, como la heparina, debido a que poseen más afinidad a los GAGs. Considerando la RA como prerrequisito para una normal fecundación, la eficacia del número de espermatozoides que sufren RA, se espera que influencie el potencial fecundante de un reproductor (Birck et al. 2010). Whitfield \& Parkinson (1995) destacan la importancia de establecer la relación entre la RA in vitro de los espermatozoides de semen descongelado con la fertilidad y el uso de agentes inductores, como la heparina y cómo se puede aplicar para observar alteraciones estructurales de los espermatozoides y como prueba de fertilidad de los reproductores. En respuesta al aumento de la demanda en programas de mejoramiento de empresas ganaderas por la correcta identificación de toros fértiles (Birck et al. 2010), se hacen necesarias pruebas complementarias de evaluación seminal, que permitan mejorar la eficiencia reproductiva.

Este estudio tuvo como objetivo evaluar la respuesta del semen congelado-descongelado a la inducción de la RA con heparina y su uso como prueba complementaria, para predecir la fertilidad del semen congelado-descongelado.

\section{MATERIALES Y MÉTODOS}

El estudio fue realizado en el laboratorio de Reproducción Animal de la Facultad de Medicina Veterinaria y Zootecnia, de la Universidad de Córdoba, localizado en Ciénaga de Oro, Córdoba, Colombia, con una temperatura promedio de $29^{\circ} \mathrm{C}, 80 \%$ de humedad relativa, precipitación anual de 1200 $\mathrm{mm}$, posición geográfica determinada entre los $8^{\circ} 45^{\prime} 27^{\prime \prime}$ de latitud Norte y 7553'24" de longitud Oeste. Fueron usados siete toros cebuínos (4 Brahman y 3 Gyr), sexualmente maduros. En la evaluación seminal posdescongelación, se evaluó la movilidad progresiva individual rápida (MPIR) y el porcentaje de anormalidades mayores (AP), menores (AS) y totales (AT). Para la evaluación de MIRP, se colocaron $20 \mu \mathrm{L}$ de semen sobre un portaobjetos y se cubrió con una laminilla, para posterior observación en el microscopio, con objetivo de 40X. La morfología espermática fue evaluada sobre un extendido en portaobjeto, a partir de la mezcla de $20 \mu \mathrm{L}$ de semen y $20 \mu \mathrm{L}$ de eosina. Para ello, se contaron 200 espermatozoides en el microscopio, con un objetivo de 100X, anotando las diferentes anormalidades encontradas. Para el proceso de inducción de RA, se aplicóp la metodología propuesta por Whitfield \& Parkinson (1992). El semen fue descongelado a $37^{\circ} \mathrm{C}$ en baño maría, vertido en un tubo cónico de $15 \mathrm{~mL}$ y centrifugado a 320 gravedades (g), durante 10 minutos; luego, el sobrenadante se descartó y los espermatozoides se resuspendieron en $5 \mathrm{~mL}$ de solución TALP y, nuevamente, se centrifugó por 10 minutos, a $320 \mathrm{~g}$. El sobrenadante fue descartado y al precipitado, se le adicionaron $0,5 \mathrm{~mL}$ de solución TALP. Para obtener una concentración final de espermatozoides $20 \mathrm{x}$ $10^{6} / \mathrm{mL}$, se realizó una dilución 1:40 de la solución anterior con agua destilada y se contaron los espermatozoides en cámara de Neubauer. La solución obtenida (Spz + TALP) fue repartida en cuatro viales de $1 \mathrm{~mL}$ cada uno, agregando $10 \mu \mathrm{g}$ de heparina/vial a dos viales, mientras que los restantes, sin heparina, se consideraron como controles. Inmediatamente, todos los tubos fueron incubados a $37^{\circ} \mathrm{C}$ 
durante una hora y cumplido este período, se adicionaron $50 \mu \mathrm{L}$ de formalina $10 \%$, a cada una de las muestras. Para la lectura, se realizaron extendidos en portaobjetos, a partir de la mezcla de $20 \mu \mathrm{L}$ de la solución anterior, con $20 \mu \mathrm{L}$ de eosina-nigrosina. La respuesta a la inducción de la RA, se valoró en microscopía de luz, con objetivo de 100x, teniendo en cuenta la diferencia de los porcentajes obtenidos de las muestras incubadas con heparina y las muestras control y expresando los resultados, de acuerdo a una escala de 0 a $100 \%$. En el análisis estadístico fue usado el programa SAEG9.1 (UFV, 2007), siendo obtenidas las medias y la desviación estándar para todas las variables y fueron realizados las pruebas de Lilliefors y Cochran y Bartlett para verificar la normalidad de los datos y la homogeneidad de las varianzas, respectivamente, no siendo necesaria la conversión de los datos de ningún parámetro estudiado. El análisis de varianza fue realizado para verificar el efecto entre raza, toro y dosis de heparina y cuando mostraron efecto significativo por el test $\mathrm{F}$, se realizó la comparación de medias por la prueba de Tukey, con 5\% de probabilidad de error. Para el análisis de las tasas de no retorno (TNR), los datos fueron agrupados en tablas de contingencia y, posteriormente, evaluados por el análisis de Chi-cuadrado, con probabilidad de 5\% de error.

\section{RESULTADOS Y DISCUSIÓN}

Los valores medios obtenidos de MPIR, AP y AT para los toros de raza Brahman y Gyr usados en este estudio fueron $77,1 \pm 2,5 \%, 0,42 \pm 0,6 \%$ y $17,1 \pm 3,5 \%$, respectivamente (Tabla 1). Estos resultados, se muestran satisfactorios para las características seminales posdescongelación, divulgadas por la Society for Theriogenology (Chenoweth et al. 1993) y el Colegio Brasileiro de Reprodução Animal (Henry \& Neves, 1998), siendo animales maduros sexualmente y usados rutinariamente como reproductores, aptos para congelación de semen. En el análisis de los datos, no se observó diferencias estadísticas entre las dosis seminales de ambas razas para estas características ( $p>0.05$ ), con excepción de las AP, donde la raza Brahman presentó menor porcentaje de defectos que la raza Gyr ( $\mathrm{p}<0.05)$. Palmieri et al. (2004) usaron semen fresco de toros criollos Romosinuano y Costeño con cuernos y no hallaron diferencias estadísticas para la MPIR y las AT, entre ambas razas ( $>>0.05)$. Siqueira et al. (2007), evaluando la calidad seminal del semen congeladodescongelado de toros Nelore, encontraron valores menores para MPIR (53,38 $\pm 11,9 \%)$, y superiores en AP de $12,48 \pm$ $5,7 \%$ y AT de 17,44 $\pm 6,08 \%$ comparados con los resultados de este estudio. Estos autores resaltan que los resultados de evaluaciones macroscópicas dependen de varios factores, como el medio utilizado para diluir el semen, las tasas de dilución, la temperatura y el tiempo de realización, durante la evaluación. Sumado a lo anterior, es necesario considerar que el espermatozoide es una célula compleja, que envuelve factores bioquímicos y morfológicos y que se puede tornar no funcional, cuando alguno de ellos es afectado. Por lo anterior, para realizar un diagnóstico de la funcionalidad e integridad del espermatozoide es necesaria la combinación de varios factores en un análisis multifactorial (Melo \& Henry, 1999), a pesar de que la evaluación convencional contempla características más fáciles de evaluar, como la movilidad, la morfología y la concentración espermática.

La inducción de RA, como técnica in vitro de evaluación de calidad seminal, contribuye a los exámenes tradicionales, al considerar características morfológicas y funcionales de los espermatozoides (Obando \& Holguín, 2003). En este estudio, la respuesta a la inducción de la RA en toros Brahman y Gyr, usando concentración de heparina de $10 \mu \mathrm{g}$, fue elevada $(\mathrm{p}<0.05)$, al considerar la diferencia con las muestras que fueron incubadas sin heparina (control), mostrando el efecto del inductor utilizado. El resultado obtenido de porcentaje de RA, se muestra inferior a los observados por Palmieri et al. (2004) y Whitfield \& Parkinson (1995) (Tabla 1). Estos resultados, se pueden deber a que en los procedimientos utilizados por estos autores, las muestras fueron incubadas con heparina, durante un período de seis horas, ya que de acuerdo con Parrish et al. (1988), un mayor tiempo de incubación de los espermatozoides

Tabla 1. Movilidad progresiva individual rápida y morfología espermática del semen postdescongelación, según raza.

\begin{tabular}{|c|c|c|c|}
\hline Variable & BRAHMAN $(\mathrm{X} \pm \mathrm{DE})$ & GYR $(\mathrm{X} \pm \mathrm{DE})$ & Media General \\
\hline MPIR & $76,9 \pm 2,5^{\mathrm{a}}$ & $77,5 \pm 2,6^{\mathrm{a}}$ & $77,1 \pm 2,5$ \\
\hline AS & $17,1 \pm 3,2^{\mathrm{a}}$ & $16,1 \pm 3,5^{\mathrm{a}}$ & $16,7 \pm 3,3$ \\
\hline AP & $0,1 \pm 0,3^{\mathrm{a}}$ & $0,8 \pm 0,7^{\mathrm{b}}$ & $0,42 \pm 0,6$ \\
\hline AT & $17,2 \pm 3,1^{\mathrm{a}}$ & $17,0 \pm 3,9^{\mathrm{a}}$ & $17,1 \pm 3,5$ \\
\hline \%RA & $85,7 \pm 4,9^{\mathrm{a}}$ & $81,7 \pm 7,3^{\mathrm{a}}$ & $83,7 \pm 6,1$ \\
\hline
\end{tabular}

${ }^{\mathrm{a}, \mathrm{b}}$ Medias con diferentes letras en la misma columna difirieron (Tukey, $\mathrm{p}<0.05$ ); MPIR: Movilidad individual rápida progresiva; AS: anormalidades menores; AP: anormalidades mayores; AT: anormalidades totales. 
con heparina, incrementa la tasa de fecundación. Obando \& Holguín (2003), al trabajar con toros de raza Brahman y Sanmartinero, al igual que Siqueira et al. (2007) con toros de raza Nelore, reportan resultados inferiores de 32,8\% y $9,85 \%$, respectivamente, valores bajos que se pueden deber que no se utilizó ningún inductor. Costa et al. (2010), usaron heparina y lisofosfaditilcolina, como inductores de RA, como parámetro para estimar la producción in vitro (PIV) de embriones Nelore y observaron que los tratamientos en los que usaron heparina mostraron mayor porcentaje de espermatozoides vivos reaccionados que los tratamientos con lisofosfatidilcolina y control, demostrando ser un eficiente inductor de RA $(\mathrm{P}<0.01)$. En un estudio realizado por Januskauskas et al. (2000), la heparina fue comparada con otro GAG, el ácido hialurónico, para evaluar el potencial fecundante de semen congelado-descongelado de toros Sueco Rojo y Blanco, observando que la heparina incrementó la frecuencia de capacitación y RA en los espermatozoides, mientras que el ácido hialurónico no tuvo ningún efecto capacitante.

En la tabla 2, se observan los valores obtenidos para las TNR, los cuales, fueron de $79,4 \pm 1,4 \%$ y $72,6 \pm 8,9 \%$, para las razas Brahman y Gyr, respectivamente, sin hallar diferencia entre las medias para ambas razas ( $p>0.05)$. En el análisis de correlaciones no se encontró una asociación entre la MPIR y las TNR, al igual que la asociación entre el \%RA y TNR; entre las AT y las TNR, se observó correlación negativa $(-0,29$; $p>0,05)$. Freneau (2011) destaca la morfología espermática como factor rutinario más importante en la evaluación de un toro, por su relación con la fertilidad, además de estar relacionada con los procesos de pubertad y de madurez sexual, lo que no coincide con nuestros resultados, debido a la variabilidad de factores que influyen en la fertilidad. Los valores de TNR de los toros Brahman y Gyr de este estudio fueron superiores a los obtenidos por Whitfield \& Parkinson (1992), quienes reportan valores entre 62,1 y $76,1 \%$ de TNR, a los 90 días, utilizando semen congelado-descongelado de toros Holstein; así mismo Siqueira et al. (2007) encontraron $63,8 \% \pm 13,7$ de tasa de gestación con toros Nelore criados a campo.

Correa et al. (1997) demuestran que no existe diferencia entre la MPIR de semen congelado-descongelado de toros con alta y baja fertilidad, similar a los resultados de este estudio, donde no se halló correlación entre los valores de MPIR y TNR. Siqueira et al. (2007) hallaron correlación negativa $(-, 22)$ con semen de toros Nelore, verificando que las muestras de semen de motilidad más alta postdescongelación no fueron las relacionadas con las tasas de preñez más altas; ello se puede dar a daños en estructuras, como el acrosoma, lo que puede representar la infertilidad de espermatozoides con alta motilidad espermática, posterior a la congelación. La correlación negativa entre las AT y las TNR obtenida $(0,29 ; p>0,05)$, es similar a la observada por Siqueira et al. (2007) $(-0,11)$, quienes trabajaron con toros Nelore. De igual forma, Correa et al. (1997), evaluando semen congelado-descongelado de toros Holstein, hallaron asociación entre el porcentaje de espermatozoides normales y la fertilidad $(0,59)$.

La asociación observada entre el porcentaje de RA y las TNR de los toros estudiados, no fue significativa ( $p>0.05)$, contrario a los hallazgos de Whitfield \& Parkinson (1992), quienes trabajando con toros de raza Holstein hallaron una correlación de $0,81 \quad(p<0.01)$. Posteriormente, estos mismos autores, usando ionóforo de calcio (A23187), como inductor, encontraron nuevamente asociación entre la RA y las TNR $(r=0.86 ; p<0.01)$ (Whitfield \& Parkinson, 1995). Los resultados obtenidos en este estudio, se pueden deber a que la tasa de gestación depende más de la influencia de otras variables externas y algunas poco controlables, como el ambiente, manejo reproductivo, alimentación, estado sanitario, reproductivo y fisiológico de las hembras, que de la reacción del acrosoma de los espermatozoides del macho cebuíno en trópico bajo. Es de anotar que aunque no se evidenció asociación entre las TNR y los porcentajes de inducción de RA en los toros Brahman y Gyr de este estudio, se observa que la heparina se comporta como un excelente inductor, permitiendo respaldar las pruebas de uso rutinario en los laboratorios de evaluación de semen congelado-descongelado. Según Salvador et al. (2001), existe la posibilidad que los estudios que evalúan el potencial de fecundación de los espermatozoides, como la inducción de RA, sean exámenes complementarios a las pruebas andrológicas de rutina, principalmente, en la selección de toros superiores o sometidos a altos desafíos de cruzamiento en campo.

Tabla 2. Tasa de no retorno al estro de las vacas sometidas a inseminación artificial con semen de los toros, de acuerdo a la raza.

\begin{tabular}{|c|c|}
\hline Raza & $\%$ TNR $(\mathrm{X} \pm \mathrm{DE})$ \\
\hline BRAHMAN & $79,4 \pm 1,4^{\mathrm{a}}$ \\
\hline GYR & $72,6 \pm 8,9^{\mathrm{a}}$ \\
\hline
\end{tabular}

TNR: Tasa de no retorno al estro; X: media; DE: desvío estándar. ${ }^{a}=$ Medias seguidas por letras minúsculas iguales en la misma columna no difieren entre sí $(\mathrm{P}<0.05)$ por el test de Chicuadrado. 
Es así, como los resultados del presente estudio demuestran que la técnica in vitro de inducción de reacción acrosómica con heparina puede ser empleada como una prueba complementaria en la evaluación de rutina del semen congelado bovino; sin embargo, de forma aislada no es eficaz en predecir el potencial fecundante del semen criopreservado.

Agradecimientos. Con gran estimación, los autores manifiestan los agradecimientos a la facultad de Medicina Veterinaria y Zootecnia de la Universidad de Córdoba y a la firma comercial Abastecedora. Conflictos de intereses: El manuscrito fue preparado y revisado con la participación de todos los autores, quienes declaramos que no existe ningún conflicto de intereses que ponga en riesgo la validez de los resultados presentados.

\section{BIBLIOGRAFÍA}

1. AX, R.L.; LENZ, R.W. 1986. Glycosaminoglycans as probes to monitor differences in fertility of bulls. J. Dairy Sci. 70(7):1477-1486.

2. BIRCK, A.; CHRISTENSEN, P.; LABOURIAU, R.; PEDERSEN, J.; BORCHERSEN, S. 2010. In vitro induction of the acrosome reaction in bull sperm and the relationship to field fertility using low-dose inseminations. Theriogenology 73:1180-1191.

3. BISSONNETTE, N.; LE' VESQUEE-SERGERIE, J.P.; THIBAULT, C.; BOISSONNEAULT, G. 2009. Spermatozoal transcriptome profiling for bull sperm motility: a potential tool to evaluate semen quality. Reproduction 138:65-80.

4. CHENOWETH, P.J.; HOPKINS, F.M.; SPITZER, J.C.; LARSEN, R.E. 1993. Guidelines for using the bull breeding soundness evaluation form. In: Theriogenology Handbook. p.B-10.

5. CORREA, J.R.; PACE, M.; ZAVOS, P. 1997. Relationships among frozen-thawed sperm characteristics assessed via the routine semen analysis, sperm functional tests and fertility of bulls in an artificial insemination program. Theriogenology. 48:721-731.

6. COSTA, M.Z.; OLIVEIRA, L.Z.; RESENDE, M.V.; LUCIO, A.C.; PERINI, A.P.; MIGUEL, M.C.V.; LIMA, V.F.M.H. 2010. Induction of the acrosome reaction test to in vitro estimate embryo production in Nelore cattle. Arq. Bras. Med. Vet. Zootec. 62 (4):771-777.

7. FRENEAU, G.E. 2011. Aspectos da morfologia espermática em touros. Rev. Bras. Reprod. Anim. 35, (2):160-170.
8. GLIEDT, D.W; ROSENKRANS, C.F; RORIE, R.W.Jr.; RAKES, J.M. 1996. Effects of oocyte maturation length, sperm capacitation time, and heparin on bovine embryo development. J Dairy Sci. 79:532535.

9. HAMMERSTEDT, R.H.; GRAHAM, J.K.; NOLAN, J.P. 1990. Cryopreservation of mammalian sperm: What we ask them to survive. J. Androl. 11:73-88.

10. HENRY, M.; NEVES, J. Eds. 1998. Manual para exame andrológico e avaliação de sêmen animal. 2.ed. Belo Horizonte: Colégio Brasileiro de Reprodução Animal. 49p.

11. JANUSKAUSKAS, A.; GIL, J.; SO"DERQUIST, L.; RODRIGUEZ-MARTINEZ, H. 2000. Relationship between sperm response to glycosami-noglycans in vitro and non-return rates of Swedish dairy AI bulls. Reprod Dom Anim. 35 (5):207-212.

12. MELO, M.; HENRY, M. 1999. Teste hiposmótico na avaliação de sêmen eqüino. Arq. Bras. Med. Vet. Zootec. 51(1):71-78.

13. MÉNDEZ, J.JR.; BURNS, P.; DE LA TORRE-SANCHEZ, J.; SEIDEL, G.JR. 2003. Effect of heparin on cleavage rates and embryo production with four bovine sperm preparation protocols. Theriogenology. 60(2):331340.

14 MILLER, D.; WINER, M.; AX, R. 1990. Heparin-binding proteins from seminal plasma bind to bovine spermatozoa and modulate capacitation by heparin. Biol.Reprod. 42:899-915.

15. OBANDO, H.; HOLGUÍN, N. 2003. Inducción de la reacción acrosómica in vitro, como prueba de fertilidad del semen bovino. ICA Informa. 30(1):4246.

16. OLIVERA, M.; RUIZ, T.; TARAZONA, A.; GIRALDO C. 2006. El espermatozoide, desde la eyaculación hasta la fertilización. Rev Col Cienc. Pec. 19(4):426-436.

17. PALMIERI, A.; SUÁREZ, D.; GONZÁLEZ, M.; ESPITIA, A. 2004. Variables seminales en toros criollos colombianos Costeño con cuernos y Romosinuano. MVZ Córdoba. 9(1):381-385.

18. PARRISH, J.; SUSKO-PARRISH, J.; WINER, M.; FIRST, N. 1988. Capacitation of bovine sperm by heparin. Biol. Reprod. 38:1171-1180. 
19. RISOPATRÓN, J.; CATALÁN, S.; SEPÚLVEDA, N.; SÁNCHEZ, R. 2005. Efecto de diferentes concentraciones de heparina sobre la capacitación espermática in vitro en canino. Rev. Cient. Vet. FCVLUZ, 16:6.

20. RODRÍGUEZ-MARTÍNEZ, H.; LARSSON, B.; ZHANG, B.R.; SÖDERQUIST, L. 1997. In vitro assessment of viability and fertilizing capacity of bull spermatozoa. J. Reprod. Develop. 43:1-11.

21. SALVADOR, D.; ANDRADE, V.; VALE FILHO, V. 2001. Potencial das proteínas do plasma seminal ou ligadas à membrana espermática como indicador da fertilidade de touros. Caderno Técnico de Veterinária e Zootecnia. 35:61-71.

22. SIQUEIRA, J.; GUIMARÃES, J.; DA COSTA, P.; HENRY, M.; TORRES, C.; SILVA, M.; SILVEIRA, T. 2007. Relação da taxa de gestação com sêmen bovino congelado e testes de avaliação espermática in vitro. Rev. Bras. Zoot. 36 (2):387-395.

23. SUTKEVICIENE, N.; RISKEVICIENE, V.; JANUSKAUSKAS, A.; ZILINSKAS, H.; ANDERSSON, M. 2009. Assessment of sperm quality traits in relation to fertility in boar semen. Acta Vet. Scand. 51:53.
24. UINIVERSIDAD FEDERAL DE VIÇOSA - UFV. 2007. Sistemas de Análisis Estadísticos y Genéticos SAEG. (Versión 9.1). Viçosa, Brasil.

25. WHITFIELD, C.; PARKINSON, T. 1995. Assessment of the fertilizing potential of frozen bovine spermatozoa by in vitro induction of acrosome reactions with calcium ionophore (A23187). Theriogenology. 44:413-422.

26. WHITFIELD, C.; PARKINSON, T. 1992. Relationship between fertility of bovine semen and In vitro induction of acrosome reactions by heparin. Theriogenology. 38:11-20.

27. YANAGIMACHI, R. 1994. Mammalian fertilization. En: Knobil, E.; Neill, J. (Eds). The physiology of reproduction. Ed. Raven Press (New York). p.189317.

28. YANG, X.; JIANG, S.; FOOTE, R.H. 1993. Bovine oocyte development following different oocyte maturation and sperm capacitation procedures. Mol. Reprod. Develop. 34(1):94-100.

Recibido: Junio 27 de 2012

Aceptado: Octubre 4 de 2012 